\title{
Correlative Imaging of Melanosoms with Ptychography, X-ray Fluorescence and Light Microscopy.
}

Gerald Falkenberg ${ }^{1,}$, Gerta Fleissner ${ }^{2}$, Philipp Alraun ${ }^{1}$, Juliane Reinhardt ${ }^{1}$, Maria Scholz ${ }^{1}$, Andreas Schropp $^{1}$, Kathryn Spiers ${ }^{1}$ Jan Garrevoet ${ }^{1}$, Christian G Schroer $^{1}$ and Günther Fleissner ${ }^{2}$

${ }^{1 .}$ Photon Science at Deutsches Elektronen-Synchrotron DESY, Hamburg, Germany.

2. Institute Cell Biology and Neuroscience, Goethe University Frankfurt/M., Germany.

* Corresponding author, Gerald.Falkenberg@ desy.de

The nature of retinal shielding pigment in the larval eyes of the midge Clunio marinus was studied by high resolution scanning X-ray fluorescence (XRF) analysis correlated with light microscopy and scanning coherent X-ray diffractive imaging (Ptychography). Clunio is known as a unique model system to decipher mechanisms underlying the complex moon-related rhythms. Shielding pigment granules within the photoreceptor cells of their primitive eyes (ocelli) have been shown to change appearance in the same rhythm [1]. The larvae are equipped with simple pigment-cup eyes (ocelli) in which the dense shielding pigment permits light access to the photosensitive rhabdomers only through a narrow pinhole resulting in a diffuse image of the environment. The pigment cup of these eyes becomes transparent during full moon [2]. Within the photoreceptor cells of the ocelli, melanin containing melanosomes form shielding pigment granules. Melanin is a nearly universally occurring pigment in different organisms and organ systems with several known functions; not only as shield against bright light (especially UVlight), but also as reservoir or sink of excessive amounts of metals, and as a component of the immune system [3]. Trace metals have been shown to be indicators of certain steps of melanogenesis. The aim of this study is to combine different non-destructive microscopy techniques to analyse the process of melanogenesis with single melanin granule-resolution.

The X-ray measurements were performed at the nanoprobe endstation (PtyNAMI) of beamline P06 at the synchrotron light source PETRA III at DESY in Hamburg (Germany) [4]. A focus size of $125 \times 130$ $\mathrm{nm}^{2}$ was obtained using nanofocusing refractive X-ray lenses (NFL) in high coherence mode for ptychography at a flux of $8 \times 10^{7}$ photons/s at a photon energy of $15 \mathrm{keV}$. With insertion of an additional prefocusing compound refractive lens (CRL) upstream in the optics hutch, a photon flux of about $2 \times 10^{9}$ photons/s was generated. However, prefocusing was only used for the XRF measurements due to reduced coherence conditions by accepting more than the coherent part of the beam, which are suboptimal for high-resolution ptychography. The coherent scatter signal was recorded for ptychography experiments using an Eiger X 4M 2D-area detector at a distance of $2.1 \mathrm{~m}$. Recently, the experimental conditions were extended by the application of Beryllium compound refractive lenses in combination with a corrective phase plate. Focus sizes below $100 \mathrm{~nm}$ at a coherent flux of $3 \times 10^{8}$ photons/s was used for Ptychographic imaging of another Clunio ocellus [5] (not shown).

We compared the relative amount of trace metals ( $\mathrm{Ca}, \mathrm{Ni}, \mathrm{Zn}, \mathrm{Cu}$ and $\mathrm{Pb}$ ), obtained by $\mathrm{XRF}$, within single melanosomes (diameter about $500 \mathrm{~nm}$ ) and the surrounding photoreceptor cells with their optical density in the light microscope and the electron density derived from the ptychography measurements (see Fig. 1). Individual pigment granules can be identified and correlated in images of all three techniques. The optical density, electron density and in particular the element concentrations differ from granule to granule with only weak correlation. We assume that the state of melanogenesis is not synchronized precisely for all granules inside the ocellus. 
In conclusion, correlative measurements with light microscopy, nanoscopic X-ray fluorescence and scanning coherent diffractive imaging (ptychography) are feasible at PETRA III beamline P06 on chemically fixed thin sections of biological tissue with nanometer scale resolution. The interplay of the image contrasts optical density, trace element concentration and electron density on the basis of individual melanosomes within the cell and tissue environment of a Clunio larva ocellus reflect the complexity of the melanization process. The ptychography measurements support the model of loading and unloading of previously empty vesicles for melanogenesis $[1,6]$.
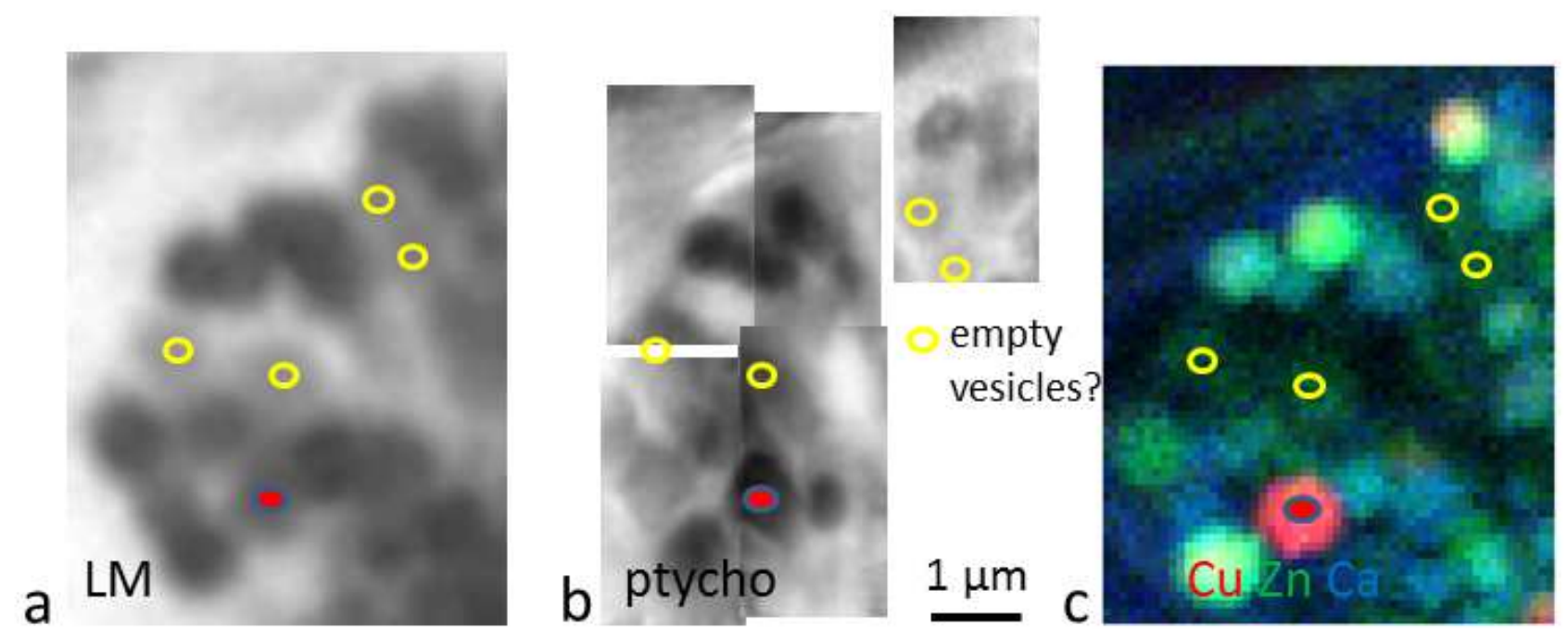

Figure 1. Clunio larva thin section in the region of an ocellus. Light microscopy (a), ptychographic image reconstruction (b), and XRF element maps (c), shown as additive combination of $\mathrm{Cu}$ (red), $\mathrm{Zn}$ (green), and $\mathrm{Ca}$ (blue). The ptychography measurement is composed of five consecutively scanned areas which are shifted from regular positions due to positional drift. The pigment granules can be identified in images of all three techniques. However, the properties optical density, electron density and in particular element concentration of individual granules differ from granule to granule with only weak correlation. Yellow circles indicate positions of (potential) empty vesicles and the red dot marks a granule with high copper concentration and electron density. (The figure is by the author(s) under a CC BY license.)

\section{References:}

[1] G Fleissner et al., Chronobiology International 25(1) (2008) 17-30.

[2] G Falkenberg et al., J. Phys., Conf. Ser. 463 (2013) 012016.

[3] F Solano, New Journal of Science 2014 (2014) 498276.

[4] CG Schroer et al., Proc. SPIE 10389 (2017) 103890E.

[5] A Schropp et al., contribution to the XRM 2018 conference.

[6] This work is supported by the German Ministry of Education and Research (BMBF) under Grant Number 05K13OD4. Beamtime at beamline P06 at PETRA III was granted within the in-house research program of DESY, a member of the Helmholtz-Association. 